GSA Data Repository Item 2016255

Eyster, A.E., Fu, R.R., Strauss, J.V., Weiss, B.P., Roots, C.F., Halverson, G.P., Evans, D.A.D., and Macdonald, F.A., 2016, Paleomagnetic evidence for a large rotation of the Yukon Block relative to Laurentia: Implications for a low-latitude Sturtian Glaciation and the break-up of Rodinia: GSA Bulletin, doi:10.1130/B31425.1.

\begin{tabular}{|l|l|l|l|l|}
\hline \multicolumn{5}{|c|}{$\begin{array}{l}\text { SUPPLEMENTARY TABLE DR1. } \\
\text { SAMPLE LOCATION DATA }\end{array}$} \\
\hline $\begin{array}{l}\text { Site/ } \\
\text { Section }\end{array}$ & MHV member & type & & Latitude $\left({ }^{\circ} \mathrm{N}\right)$ \\
\hline A1117 & dike & site & 64.73438 & -139.76778 \\
\hline A1118 & sill & site & 64.73637 & -139.76372 \\
\hline A1119 & dike feeder & site & 64.73637 & -139.76372 \\
\hline A1120 & dike & site & 64.68373 & -139.86320 \\
\hline A1121 & dike & site & 64.68373 & -139.86320 \\
\hline A1122 & Seela Pass Fm. & 5.6 m section & 64.68060 & -139.96107 \\
\hline A1123 & Member A & 49.0 m section & 64.68060 & -139.96107 \\
\hline A1125 & Member A & 21.9 m section & 64.76650 & -140.11252 \\
\hline A1126 & Eagle Creek fm. (with basalt clasts) & conglomerate test/ & 64.76176 & -140.12360 \\
& & 22 m section & & \\
\hline A1127 & dike & site & 64.76179 & -140.12360 \\
\hline A1128 & Member B & 191.5 m section & 64.70884 & -140.04704 \\
\hline A1129 & Eagle Creek fm. (with basalt clasts) & conglomerate test & 64.70535 & -140.04903 \\
\hline A1201 & Member D-younger intrusion & site & 64.66237 & -140.01630 \\
\hline A1202 & Member D- younger intrusion & site & 64.66253 & -140.00642 \\
\hline A1203 & Member D & 1.3 m section & 64.66323 & -140.00482 \\
\hline A1204 & Member C & 3 m section & 64.28337 & -140.82295 \\
\hline A1206 & Member B & site & 64.65967 & -140.00342 \\
\hline A1207 & Member D & site & 64.28337 & -140.82295 \\
\hline A1209 & Member B and Eagle Creek fm. & 273.0 m section & 64.67632 & -139.86698 \\
\hline A1210 & Member A & 55.4 m section & 64.68047 & -140.86070 \\
\hline
\end{tabular}




\section{Sample Analysis}

If a specimen component fit had a MAD $>15$, it was discarded from subsequent analysis. For samples with multiple specimens with the same component I used Fisher statistics to find an average component direction for the sample. The data was discarded if the associated CSD $>20$. In some cases, if there were two specimens, one that seemed very different from all the other samples, and the other that was similar, then I would examine that specimen that was an outlier and exclude it from the mean if it seemed anomalous (unblocking temperatures, etc)

Finally, as this dataset was particularly noisy, some component directions did not always display desired precision. This was particularly apparent for the LT and HT components. For the LT components, I choose to exclude some LT component directions from the site/section means if they were very shallow or appeared to be drastically anomalous ( $\sim 60$ degrees away) from many of the other samples from the site/section. For the HT components, the main difference was that some components were upper hemisphere and some were lower hemisphere directions. In my analysis I split up the means into a lower hemisphere and upper hemisphere means. I then used the cutoff of $\mathrm{N}>3$ and a95<20 for each mean. It was in this way that the HT1 and HT2 clusters became apparent and isolated.

\begin{tabular}{|c|c|c|c|c|c|c|}
\hline \multicolumn{7}{|c|}{$\begin{array}{c}\text { SUPPLEMENTARY TABLE DR2. } \\
\text { LOW-TEMPERATURE COMPONENT MEAN DIRECTIONS WITH } \alpha_{95}<20^{\circ}\end{array}$} \\
\hline Site/Section & $\begin{array}{l}\text { LT demag. } \\
\text { temp. }\end{array}$ & $\begin{array}{l}\mathrm{D} / \mathrm{l} \text { (in situ) } \\
\left({ }^{\circ}\right)\end{array}$ & $\begin{array}{l}\text { D/I (tilt corrected) } \\
\left({ }^{\circ}\right)\end{array}$ & $\begin{array}{l}\alpha_{95} \\
\left({ }^{\circ}\right)\end{array}$ & $\mathrm{k}$ & $\mathrm{n} / \mathrm{n}_{\mathrm{c}}$ \\
\hline \multicolumn{7}{|c|}{ Approach 1} \\
\hline A1128 & $100^{\circ} \mathrm{C}$ & $50.4 / 79.7$ & $209.0 / 82$ & 19.8 & 2.9 & $27 / 59$ \\
\hline A1210 & $\begin{array}{c}150^{\circ} \mathrm{C} \\
\left(\mathrm{LN}-250^{\circ} \mathrm{C}\right)\end{array}$ & $51.8 / 84.4$ & $212.2 / 55.3$ & 9.8 & 20.6 & $12 / 22$ \\
\hline \multicolumn{2}{|c|}{$\begin{array}{l}\text { MHV } \\
\text { Mean Direction }\end{array}$} & $\begin{array}{l}54.0 / 80.9 \\
\alpha_{95}=5.8 \\
k=1886.5 \\
C S D=1.86\end{array}$ & $\begin{array}{c}209.2 / 69.7 \\
\alpha_{95}=56.6 \\
k=21.6 \\
C S D=17.42\end{array}$ & & & $\begin{array}{l}2 \text { sections } \\
39 \text { samples }\end{array}$ \\
\hline \multicolumn{7}{|c|}{ Approach 2} \\
\hline A1210 & $\begin{array}{c}150^{\circ} \mathrm{C} \\
\left(\mathrm{LN}-250^{\circ} \mathrm{C}\right)\end{array}$ & $44.7 / 83.9$ & $213.2 / 56.1$ & 10.3 & 20.4 & $\begin{array}{l}\text { 11flows } \\
12 / 22\end{array}$ \\
\hline \multicolumn{2}{|c|}{$\begin{array}{l}\text { MHV } \\
\text { Mean Direction }\end{array}$} & $\begin{array}{c}44.7 / 83.9 \\
\alpha_{95}=10.3 \\
k=20.4\end{array}$ & $\begin{array}{c}213.2 / 56.1 \\
\alpha_{95}=10.3 \\
k=20.4\end{array}$ & & & $\begin{array}{c}11 \text { flows } \\
1 \text { section } \\
12 \text { samples }\end{array}$ \\
\hline \multicolumn{7}{|c|}{ Approach 3} \\
\hline \multicolumn{7}{|c|}{ N.D. } \\
\hline \multicolumn{7}{|c|}{$\begin{array}{l}\mathrm{D} \text { and } \mathrm{I}-\text { mean declination and inclination } \\
\mathrm{k}-\text { Fisher's (1953) precision parameter } \\
\alpha_{95} \text { - radius of confidence circle for the mean direction } \\
n-\text { number of samples used }\end{array}$} \\
\hline
\end{tabular}




\begin{tabular}{|c|c|c|c|c|c|c|}
\hline \multicolumn{7}{|c|}{$\begin{array}{c}\text { SUPPLEMENTARY TABLE DR3. } \\
\text { MID-TEMPERATURE COMPONENT DIRECTIONS } \alpha_{95}<20^{\circ}\end{array}$} \\
\hline $\begin{array}{l}\text { Site/ } \\
\text { Section }\end{array}$ & $\begin{array}{l}\text { MT demag. temp. } \\
\left({ }^{\circ} \mathrm{C}\right)\end{array}$ & $\mathrm{D} / \mathrm{I}$ (in situ) $\left({ }^{\circ}\right)$ & D/I (tilt corrected) $\left({ }^{\circ}\right)$ & $\alpha_{95}\left(^{\circ}\right)$ & $\mathrm{k}$ & $\mathrm{n} / \mathrm{n}_{\mathrm{c}}$ \\
\hline \multicolumn{7}{|c|}{ Approach 1} \\
\hline A1123 & $425(300-500)$ & $145.4 /-81.5$ & $50.3 /-51.5$ & 7.3 & 28.6 & $15 / 17$ \\
\hline A1125 & $450(375-515)$ & $67.2 /-88.0$ & $62.4 /-36.1$ & 5.0 & 128.2 & $7 / 11$ \\
\hline A1126 & $450(350-475)$ & $225.6 /-86.2$ & $14.5 /-54.8$ & 9.3 & 31.7 & $8 / 10$ \\
\hline A1128 & $475(200-530)$ & $160.6 /-84.0$ & $60.2 /-74.1$ & 4.1 & 24.5 & $52 / 59$ \\
\hline A1203 & $450(415-475)$ & 196.6/-78.8 & 190.8/-70.9 & 4.8 & 135.7 & $6 / 7$ \\
\hline A1204 & $425(450-530)$ & $167.4 /-64.7$ & $218.1 /-75.5$ & 14.9 & 27.3 & $5 / 8$ \\
\hline A1209 & $425(425-560)$ & $145.0 /-82.8$ & $35.8 /-45.6$ & 8.9 & 19.4 & $15 / 21$ \\
\hline A1210 & $475(400-545)$ & $120.1 /-81.4$ & $48.1 /-48.5$ & 2.8 & 155.9 & $19 / 22$ \\
\hline $\begin{array}{l}\text { MHV Mea } \\
\text { Direction }\end{array}$ & & $\begin{array}{c}161.7 /-82.1 \\
\alpha_{95}=5.9 \\
k=89.6\end{array}$ & $\begin{array}{c}49.1 /-65.4 \\
\alpha_{95}=20.1 \\
k=9.5\end{array}$ & & & $\begin{array}{l}8 \text { sections } \\
128 \text { samples }\end{array}$ \\
\hline \multicolumn{7}{|c|}{ Approach 2} \\
\hline A1123 & $425(300-500)$ & $147.5 /-82.2$ & $49.1 /-51.7$ & 7.6 & 28.4 & $\begin{array}{l}14 \text { flows } \\
15 / 17\end{array}$ \\
\hline A1125 & $450(375-515)$ & $67.2 /-88.0$ & $62.4 /-36.1$ & 5.0 & 128.2 & $\begin{array}{c}7 \text { flows } \\
7 / 11\end{array}$ \\
\hline A1126 & $450(350-475)$ & $225.6 /-86.2$ & $14.5 /-54.8$ & 9.3 & 31.7 & $8 / 10$ \\
\hline A1128 & $475(200-530)$ & $176.4 /-84.7$ & $58.9 /-77.9$ & 6.5 & 40.9 & $\begin{array}{l}13 \text { flows } \\
45 / 59\end{array}$ \\
\hline A1203 & $450(415-475)$ & $196.7 /-78.8$ & $190.8 /-70.9$ & 8.6 & 845 & $\begin{array}{l}2 \text { flows } \\
6 / 7\end{array}$ \\
\hline A1204 & $425(450-530)$ & $171.5 /-63.2$ & $217.9 /-73.5$ & $\begin{array}{c}49.4 \\
\mathrm{CSD}=15.4\end{array}$ & 27.7 & $\begin{array}{l}2 \text { flows } \\
5 / 8\end{array}$ \\
\hline A1209 & $425(425-560)$ & $145.6 /-83.8$ & $34.5 /-45.2$ & 9.2 & 19.5 & $\begin{array}{c}14 \text { flow } \\
15 / 21\end{array}$ \\
\hline A1210 & $475(400-545)$ & $118.5 /-81.3$ & $48.1 /-48.3$ & 3.3 & 160.0 & $\begin{array}{l}14 \text { flows } \\
19 / 22\end{array}$ \\
\hline $\begin{array}{l}\text { MHV Mea } \\
\text { Direction }\end{array}$ & & $\begin{array}{c}164.4 / 82.3 \\
\alpha_{95}=6.3 \\
k=79.4\end{array}$ & $\begin{array}{c}48.4 /-66.0 \\
\alpha_{95}=20.6 \\
k=8.2\end{array}$ & & & $\begin{array}{c}1 \text { site } \\
7 \text { sections } \\
74 \text { stratigraphic } \\
\text { horizons } \\
121 \text { samples } \\
\end{array}$ \\
\hline \multicolumn{7}{|c|}{ Approach 3} \\
\hline $\begin{array}{l}\text { A1128 } \\
\text { Flow } 1\end{array}$ & $475(200-530)$ & $87.2 /-66.2$ & $134.2 /-73.0$ & 6.9 & 316.8 & $3 / 3$ \\
\hline $\begin{array}{l}\text { A1128 } \\
\text { Flow } 2\end{array}$ & $475(200-530)$ & 207.1/-79.6 & $58.4 /-81.8$ & 12.2 & 102 & $3 / 4$ \\
\hline $\begin{array}{l}\text { A1128 } \\
\text { Flow } 2\end{array}$ & $475(200-530)$ & 169.9/-73.1 & $102.6 /-75.1$ & 11.3 & 24.8 & $8 / 9$ \\
\hline $\begin{array}{l}\text { A1128 } \\
\text { Flow } 3\end{array}$ & $475(200-530)$ & $213.4 /-89.5$ & $41.3 /-72.5$ & 4.9 & 55.7 & $16 / 18$ \\
\hline $\begin{array}{l}\text { A1203 } \\
\text { Flow } 1\end{array}$ & $450(415-475)$ & $189.1 /-80.2$ & $186.4 /-72.1$ & 10 & 150.9 & $3 / 3$ \\
\hline $\begin{array}{l}\text { A1203 } \\
\text { Flow } 2\end{array}$ & $450(415-475)$ & $202.6 /-77.3$ & 194.8/-69.6 & 12.7 & 94.8 & $3 / 4$ \\
\hline $\begin{array}{l}\text { A1204 } \\
\text { Flow } 1\end{array}$ & $425(450-530)$ & 148.3/-69.0 & $220 /-84.4$ & 12.2 & 102.4 & $3 / 6$ \\
\hline $\begin{array}{l}\text { A1210 } \\
\text { Flow } 1\end{array}$ & $475(400-545)$ & $124.2 /-82.2$ & $47.1 /-49.2$ & 12.3 & 101.8 & $3 / 4$ \\
\hline
\end{tabular}




\begin{tabular}{|c|c|c|c|c|c|c|}
\hline \multicolumn{7}{|c|}{$\begin{array}{l}\text { SUPPLEMENTARY TABLE DR4. } \\
\text { HIGH-TEMPERATURE HT2 COMPONENT DIRECTIO }\end{array}$} \\
\hline $\begin{array}{l}\text { Sitel } \\
\text { Section }\end{array}$ & $\begin{array}{l}\text { HT2 demag. } \\
\text { temp. }\left({ }^{\circ} \mathrm{C}\right)\end{array}$ & $\begin{array}{c}\mathrm{D} / \mathrm{I} \\
\left(\text { in situ) }\left({ }^{\circ}\right)\right.\end{array}$ & $\begin{array}{c}\mathrm{D} / \mathrm{I} \\
\text { (tilt corrected) } \\
\left(^{\circ}\right)\end{array}$ & $\alpha_{95}\left({ }^{\circ}\right)$ & $\mathbf{k}$ & $n / n_{c}$ \\
\hline \multicolumn{7}{|c|}{ Approach 1} \\
\hline A1123 & $565(555-575)$ & $51.6 /-51.4$ & $46.4 /-11.7$ & 18.6 & 7.0 & $11 / 17$ \\
\hline A1128 & $570(350-585)$ & $37.5 /-48.9$ & $38.3 /-30.9$ & 16.8 & 30.7 & $4 / 59$ \\
\hline A1209 & $680(655-680)$ & $61.7 /-59.1$ & $44.6 /-15.6$ & 14.36 & 74.7 & $4 / 21$ \\
\hline A1210 & $595(545-645)$ & $58.6 /-40.5$ & $52.7 /-2.8$ & 10.1 & 14.2 & $16 / 22$ \\
\hline \multicolumn{2}{|c|}{$\begin{array}{l}\text { MHV } \\
\text { Mean Direction }\end{array}$} & $\begin{array}{c}52.2 /-50.3 \\
\alpha_{95}=11.7 \\
k=62.3\end{array}$ & $\begin{array}{c}45.8 /-15.3 \\
\alpha_{95}=14.9 \\
k=39.0 \\
\end{array}$ & & & $\begin{array}{l}4 \text { sections } \\
35 \text { samples }\end{array}$ \\
\hline \multicolumn{7}{|c|}{ Approach 2} \\
\hline A1123 & $565(555-575)$ & $50.0 /-51.4$ & $45.3 /-11.5$ & 18.8 & 6.8 & $\begin{array}{c}\text { 11flows } \\
11 / 17\end{array}$ \\
\hline A1128 & $570(350-685)$ & $45.0 /-46.2$ & $44.1 /-28.2$ & $\begin{array}{c}52.3 \\
\text { CSD }= \\
16\end{array}$ & 25.0 & $\begin{array}{c}\text { 2flows } \\
4 / 59\end{array}$ \\
\hline A1209 & $680(655-680)$ & 61.7/-59.1 & $44.6 /-15.6$ & 14.36 & 74.7 & $\begin{array}{l}4 \text { flows } \\
4 / 21\end{array}$ \\
\hline A1210 & $595(545-645)$ & $60.18 /-40.3$ & $54.0 /-3.1$ & 12.2 & 15.1 & $\begin{array}{l}11 \text { flows } \\
13 / 22\end{array}$ \\
\hline \multicolumn{2}{|c|}{$\begin{array}{l}\text { MHV } \\
\text { Mean Direction }\end{array}$} & $\begin{array}{c}54.0 /-49.5 \\
\alpha_{95}=10.9 \\
k=72.3 \\
\end{array}$ & $\begin{array}{c}47.1 /-14.6 \\
\alpha_{95}=13.0 \\
k=50.6 \\
\end{array}$ & & & $\begin{array}{c}4 \text { sections } \\
28 \text { flows } \\
32 \text { samples } \\
\end{array}$ \\
\hline \multicolumn{7}{|c|}{ Approach 3} \\
\hline $\begin{array}{l}\text { A1128 } \\
\text { Flow } 1\end{array}$ & 575 & $29.3 /-51.1$ & $32.2 /-33.4$ & 16.9 & 30.65 & $3 / 3$ \\
\hline \multicolumn{2}{|c|}{$\begin{array}{l}\text { MHV } \\
\text { Mean Direction }\end{array}$} & N. D. & N.D & & & \\
\hline
\end{tabular}

$\mathrm{D}$ and $\mathrm{I}-\mathrm{D}$ and $\mathrm{I}-$ mean declination and inclination k-Fisher's (1953) precision parameter

$a_{95}$-radius of confidence circle for the mean direction

$\mathrm{n}$-number of samples used

$\mathrm{n}_{\mathrm{c}}$-total number of stratigraphic horizons or samples collected 


\begin{tabular}{|c|c|c|c|c|c|c|}
\hline \multicolumn{7}{|c|}{$\begin{array}{l}\text { SUPPLEMENTARY TABLE DR5. } \\
\text { HIGH-TEMPERATURE HT1 COMPONENT DIRECTIONS WITH } \alpha_{95}<20^{\circ}\end{array}$} \\
\hline \begin{tabular}{|c|} 
Sitel \\
Section
\end{tabular} & $\begin{array}{l}\text { HT1 demag. } \\
\text { temp. }\left({ }^{\circ} \mathrm{C}\right)\end{array}$ & $\begin{array}{c}\mathrm{D} / \mathrm{I} \\
\text { (in situ) }\left({ }^{\circ}\right)\end{array}$ & $\begin{array}{c}\mathrm{D} / \mathrm{I} \\
\text { (tilt corrected) }\left({ }^{\circ}\right)\end{array}$ & $\alpha_{95}\left({ }^{\circ}\right)$ & $\mathbf{k}$ & $\mathbf{n} / \mathbf{n}_{\mathrm{c}}$ \\
\hline \multicolumn{7}{|c|}{ Approach 1} \\
\hline $\begin{array}{l}\text { A1126 } \\
\text { matrix }\end{array}$ & $\begin{array}{c}560 \\
(545-585)\end{array}$ & $349.1 / 80.9$ & $209.1 / 59.0$ & 16.4 & 57.7 & $4^{\# / 5}$ \\
\hline $\begin{array}{l}\text { A1126 } \\
\text { clasts }^{*}\end{array}$ & $\begin{array}{c}555 \\
(530-585)\end{array}$ & $72.3 / 43.0$ & $116.4 / 52.9$ & 114.5 & 1.1 & $5 / 5$ \\
\hline A1128 & $\begin{array}{c}575 \\
(400-630)\end{array}$ & $168.5 / 60.3$ & $185.0 / 47.5$ & 4.9 & 31.2 & $28 / 59$ \\
\hline A1203 & $560-580$ & $179.1 / 34.8$ & $178.7 / 42.8$ & 10.5 & 33.9 & $7 / 7$ \\
\hline A1207 & $575-585$ & $5.3 /-21.2$ & $5.5 /-29.2$ & 13.3 & 18.3 & $9 / 9$ \\
\hline A1209 & $575-685$ & $170.9 / 74.9$ & $196.7 / 29.8$ & 12.35 & 13.3 & $12 / 21$ \\
\hline $\begin{array}{l}\text { MHV } \\
\text { Mean Dire }\end{array}$ & ction $^{\dagger}$ & $\begin{array}{c}179.1 / 58.1 \\
\alpha_{95}=31.3 \\
k=6.9\end{array}$ & $\begin{array}{c}190.0 / 42.1 \\
\alpha_{95}=14.3 \\
k=29.5\end{array}$ & & & $\begin{array}{l}1 \text { site and } 4 \text { sections } \\
60 \text { samples }\end{array}$ \\
\hline \multicolumn{7}{|c|}{ Approach 2} \\
\hline $\begin{array}{l}\text { A1126 } \\
\text { matrix }\end{array}$ & $\begin{array}{c}560 \\
(545-585)\end{array}$ & $349.1 / 80.9$ & $209.1 / 59.0$ & 16.4 & 57.7 & $\begin{array}{l}4 \text { horizons } \\
4 / 5(2 c)\end{array}$ \\
\hline $\begin{array}{l}\text { A1126 } \\
\text { clasts* }^{*}\end{array}$ & $\begin{array}{c}555 \\
(530-585)\end{array}$ & $72.3 / 43.0$ & $116.4 / 52.9$ & 114.5 & 1.1 & $5 / 5$ \\
\hline A1128 & $\begin{array}{c}575 \\
(400-630)\end{array}$ & $158.9 / 58.8$ & $177.7 / 47.8$ & 8.5 & 29.6 & $\begin{array}{l}11 \text { flows } \\
28 / 59\end{array}$ \\
\hline A1203 & $560-580$ & $179.5 / 35.0$ & $179.2 / 43.0$ & 13.1 & 367 & $\begin{array}{c}2 \text { flows } \\
7 / 7\end{array}$ \\
\hline A1207 & $575-585$ & $5.3 /-21.2$ & $5.5 /-29.2$ & 13.3 & 18.3 & $\begin{array}{c}1 \text { flow } \\
9 / 9\end{array}$ \\
\hline A1209 & $575-585$ & $149.4 / 79.5$ & $195.8 / 36.2$ & 14.8 & 11.6 & $\begin{array}{c}10 \text { flow } \\
10 / 21\end{array}$ \\
\hline \begin{tabular}{|l} 
MHV \\
Mean Dire
\end{tabular} & ction $^{\dagger}$ & $\begin{array}{c}175.6 / 59.2 \\
\alpha_{95}=32.7 \\
k=6.4\end{array}$ & $\begin{array}{c}188.4 / 43.5 \\
\alpha_{95}=13.6 \\
k=32.4\end{array}$ & & & $\begin{array}{c}1 \text { site and } 4 \text { sections } \\
28 \text { stratigraphic } \\
\text { horizons/flows } \\
58 \text { samples }\end{array}$ \\
\hline \multicolumn{7}{|c|}{ Approach 3} \\
\hline $\begin{array}{l}\text { A1203 } \\
\text { Flow } 1\end{array}$ & $560-580$ & $176.7 / 33.2$ & $176.0 / 41.1$ & 18.6 & 25.3 & $4 / 4$ \\
\hline $\begin{array}{l}\text { A1203 } \\
\text { Flow } 2\end{array}$ & $560-580$ & $182.4 / 36.9$ & $182.5 / 44.9$ & 19.7 & 40.4 & $3 / 3$ \\
\hline A1207 & $575-585$ & $5.3 /-21.2$ & $5.5 /-29.2$ & 13.3 & 18.3 & $9 / 9$ \\
\hline $\begin{array}{l}\text { A1128 } \\
\text { Flow } 1\end{array}$ & $500-575$ & $157.4 / 64.2$ & $174.8 / 54.6$ & 15.4 & 36.5 & $4 / 4$ \\
\hline $\begin{array}{l}\text { A1128 } \\
\text { Flow } 2\end{array}$ & $560-605$ & $183.9 / 60.7$ & $196.3 / 45.1$ & 8.8 & 47.9 & $7 / 9$ \\
\hline $\begin{array}{l}\text { A1128 } \\
\text { Flow } 3\end{array}$ & $575-630$ & $163.8 / 51.4$ & $178.0 / 39.7$ & 16.45 & 57.2 & $3 / 3$ \\
\hline $\begin{array}{l}\text { A1128 } \\
\text { Flow } 4\end{array}$ & $400-575$ & $156.6 / 66.6$ & $182.3 / 55.0$ & 13.0 & 50.25 & $4 / 19$ \\
\hline \multicolumn{2}{|c|}{$\begin{array}{l}\text { MHV } \\
\text { Mean Direction }\end{array}$} & $\begin{array}{c}174.9 / 48.3 \\
\alpha_{95}=14.6 \\
k=18.1\end{array}$ & $\begin{array}{c}182.4 / 44.4 \\
\alpha_{95}=7.7 \\
k=61.8\end{array}$ & & & $\begin{array}{c}7 \text { flows } \\
34 \text { samples }\end{array}$ \\
\hline
\end{tabular}

$\mathrm{D}$ and $\mathrm{I}-\mathrm{D}$ and $\mathrm{I}-$ mean declination and inclination

k-Fisher's (1953) precision parameter

$\alpha_{95}$-radius of confidence circle for the mean direction

$\mathrm{n}$-number of samples used

$\mathrm{n}_{\mathrm{c}}$-total number of stratigraphic horizons or samples collected

${ }^{*}$ Not used in mean direction

†Used reversed A1207 site mean direction in calculation

${ }^{*} 2$ of these samples are great circle fits 


\begin{tabular}{|l|l|l|l|}
\hline \multicolumn{4}{|c|}{ SUPPLEMENTARY TABLE DR6. } \\
\hline & Kent and Irving 2010 & Besse and Courtillot, 2002 & Torsvik et al., 2012 \\
& 70 Ma pole & $\begin{array}{l}\text { 77.9 Ma pole } \\
\text { 80 Ma Pole }\end{array}$ \\
\hline $\begin{array}{l}\text { MT pole } \\
\text { Post-rotation }\end{array}$ & Watson's V: 1.0 & Watson's V: 1.5 & Watson's V: 0.9 \\
& Vcritical: 7.0 & Vcritical: 6.7 & Vcritical: 6.8 \\
& PASS & PASS & PASS \\
\hline $\begin{array}{l}\text { MT pole } \\
\text { Pre-rotation }\end{array}$ & Watson's V: 7.8 & Watson's V: 6.9 & Watson's V: 8.6 \\
& Vcritical: 6.9 & Vcritical: 6.7 & Vcritical: 6.8 \\
& FAIL & FAlL & FAIL \\
\hline
\end{tabular}


SUPPLEMENTARY TABLE DR7.

\begin{tabular}{|c|c|c|c|}
\hline \multirow[t]{2}{*}{ Craton/block/terrane } & \multicolumn{2}{|c|}{ Euler Pole } & \multirow[t]{2}{*}{ Angle of Rotation $\left({ }^{\circ}\right)$} \\
\hline & Latitude $\left({ }^{\circ} \mathbf{N}\right)$ & Longitude $\left({ }^{\circ} \mathrm{E}\right)$ & \\
\hline \multicolumn{4}{|c|}{$720 \mathrm{Ma}$} \\
\hline Laurentia & -44.77 & -14.89 & 133.98 \\
\hline Yukon block* & -66.81 & 45.17 & 54.99 \\
\hline Amazonia & 1.47 & 52.28 & 110.1 \\
\hline Pampean/Rio de La Plata & 2.77 & 52.94 & 135.76 \\
\hline Sao Francisco & 9.65 & 54.38 & 129.51 \\
\hline Baltica & 45.09 & -143.91 & 177.44 \\
\hline Congo & 16.74 & 85.88 & 126.21 \\
\hline West Africa & 4.46 & 82.71 & 135.73 \\
\hline Kalahari & 22.36 & 83.48 & 122.25 \\
\hline Australia & -11.67 & -124.32 & -89.35 \\
\hline North China & 1.44 & 178.52 & -127.11 \\
\hline India & 67.52 & 70.38 & 35.07 \\
\hline Siberia & 6.01 & -18.79 & 59.41 \\
\hline Tarim & 37.04 & 122.87 & -133.4 \\
\hline \multicolumn{4}{|c|}{$615 \mathrm{Ma}$} \\
\hline Laurentia & -31.65 & 26.07 & 145.61 \\
\hline Yukon block* & 0.0 & 0.0 & 101 \\
\hline Amazonia & 15.85 & 56.36 & 143.78 \\
\hline Pampean/Rio de La Plata & 15.92 & 52.13 & 144.2 \\
\hline Sao Francisco & 11.72 & 54.46 & 137.73 \\
\hline Baltica & 33.91 & -132.86 & 175.83 \\
\hline Congo & 17.73 & 83.46 & 133.94 \\
\hline West Africa & 22.01 & 84.04 & 136.37 \\
\hline Kalahari & 19.32 & 83.66 & 133.19 \\
\hline Australia & -2.47 & -141.79 & -76.98 \\
\hline North China & 4.28 & 9.57 & 146.82 \\
\hline India & 22.3 & -58.21 & -31.33 \\
\hline Siberia & 12.44 & -176.67 & -109.38 \\
\hline Tarim & 31.22 & 108.16 & -139.28 \\
\hline
\end{tabular}

${ }^{*}$ rotated relative to Laurentia 